\title{
The Gemini High-Resolution Optical SpecTrograph (GHOST) bench spectrograph optical design
}

\author{
John Pazder ${ }^{* a}$, Greg Burley ${ }^{\mathrm{a}}$, Michael J. Ireland ${ }^{\mathrm{b}}$, Gordon Robertson ${ }^{\mathrm{c}, \mathrm{d}}$, \\ Andrew Sheinis ${ }^{\mathrm{c}}$, Ross Zhelem ${ }^{\mathrm{c}}$ \\ ${ }^{a}$ National Research Council Canada, Herzberg Astronomy and Astrophysics, 5071 West Saanich \\ Road, Victoria, B.C., Canada, V9E 2E7; \\ ${ }^{b}$ Research School of Astronomy and Astrophysics, Australian National University, Canberra, \\ ACT 2611, Australia; \\ ${ }^{c}$ Australian Astronomical Observatory, North Ryde, NSW 2113, Australia \\ ${ }^{\mathrm{d}}$ School of Physics, University of Sydney, NSW 2006, Australia
}

\begin{abstract}
Gemini High-Resolution Optical SpecTrograph (GHOST) is a fiber-fed spectrograph being developed for the Gemini telescope. GHOST is a white pupil échelle spectrograph with high efficiency and a broad continuous wavelength coverage $(363-1000 \mathrm{~nm})$ with $\mathrm{R}>50,000$ in two-object mode and $\mathrm{R}>75,000$ in single-object mode. The design incorporates a novel zero-Petzval sum white pupil relay to eliminate grating aberrations at the cross-dispersers. Cameras are based on non-achromatic designs with tilted detectors to eliminate the need for exotic glasses. This paper outlines the optical design of the bench-mounted spectrograph and the predicted spectrograph resolution and efficiency for the spectrograph.
\end{abstract}

Keywords: Gemini telescope, high resolution spectrograph, Echelle spectrograph, white pupil relay, VPH, VPHG, volume phase holographic grating

\section{INTRODUCTION}

The Gemini High-Resolution Optical SpecTrograph (GHOST) is a high resolution fiber fed échelle spectrograph being developed for the Gemini South telescope. The instrument development is a collaboration between the Australian Astronomical Observatory (AAO), Herzberg Astronomy and Astrophysics (HAA) at the National Research Council Canada, and the Australian National University (ANU). The instrument group at HAA is responsible for the design and construction of the bench spectrograph for Gemini High-Resolution Optical SpecTrograph (GHOST) instrument. This paper focuses on the details of the optical design of the bench spectrograph from the exit of the micro lens slit unit to the detector. The overall instrument design is described in a paper by Sheinis ${ }^{1}$ published in this proceeding.

GHOST is a fiber-fed spectrograph with a single object high resolution mode and a two object standard resolution mode. Micro-lens based integral field units are used to image slice a 1.2" stellar object by a factor of 5 in width using 19 fibers in the high resolution mode and by a factor of 3 using 7 fibers in the standard resolution mode. This image slicing reduces the slit-width*resolving power product required for the spectrograph at the expense of a longer slit. The fiber feed provides a significant advantage of being able to place the spectrograph in the Gemini pier lab, which greatly improves the spectrograph stability.

* john.pazder@nrc-cnrc.gc.ca

Ground-based and Airborne Instrumentation for Astronomy VI, edited by Christopher J. Evans, Luc Simard, Hideki Takami Proc. of SPIE Vol. 9908, 99087F · (C) 2016 SPIE · CCC code: 0277-786X/16/\$18 · doi: 10.1117/12.2234366 
The optical layout of the échelle spectrograph is based on a two arm asymmetric white pupil design with Volume Phase Holographic Grating (VPHG) cross dispersion, and is shown in Figure 1. The top-level optical design parameters are listed Table 1. This two arm design is possible because of the advances in detector technology - full wavelength coverage can be achieved with a $4 \mathrm{kx} 4 \mathrm{k}$ and $6 \mathrm{kx} 6 \mathrm{k}$ detector, and also because of the advances in VPHG manufacturing capabilities.

The design achieves high efficiency through the careful selection and optimization of dispersers, optics and coatings. The échelle has near theoretical diffraction efficiencies of $\sim 80 \%$ at order centers. The white pupil magnification has been selected to yield optimal VPHG cross disperser band pass and manufacturability. The white pupil relay is a zero-Petzval sum optic with near diffraction-limited performance, eliminating the need for cylindrical optics in the cameras. The newest high performance UV enhanced silver coatings are used to minimize losses from the white pupil relay. The camera designs have been optimized for superb image quality, with better than $80 \%$ ensquared energy in a pixel. Glass and coating optimization has minimized losses in the cameras, with predicted camera transmittances greater than $96 \%$ and $94 \%$ for the red and blue cameras respectively. The total predicted transmittance (from the exit of the slit unit to the detector, not including the CCD QE) for the spectrograph peaks at $62 \%$ at $450 \mathrm{~nm}$, is $47 \%$ at $363 \mathrm{~nm}$ and has an average of over $52 \%$ from $363 \mathrm{~nm}$ to $1000 \mathrm{~nm}$.

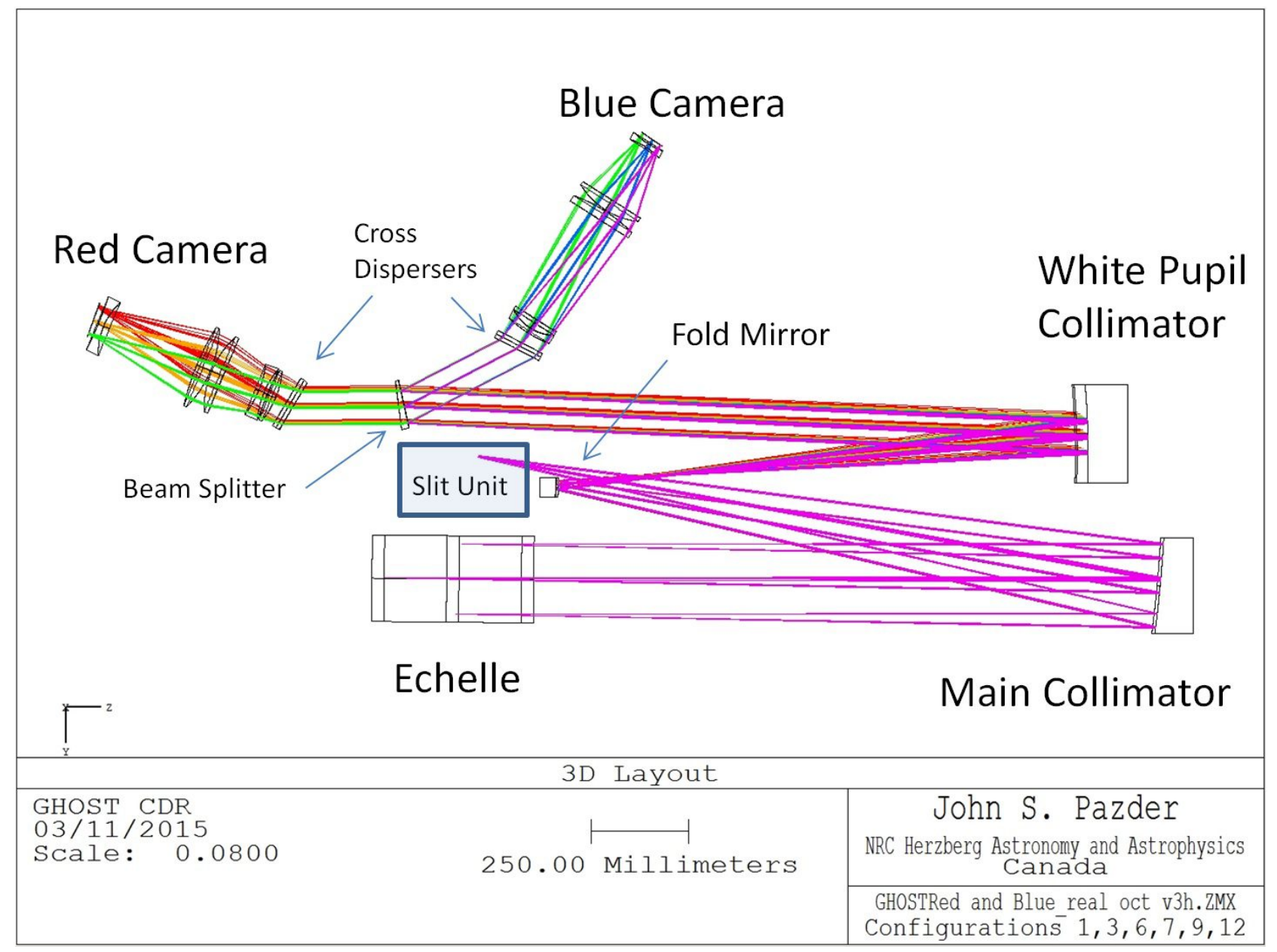

Figure 1. GHOST spectrograph optical layout 
Table 1. Top-level spectrograph design parameters.

\begin{tabular}{|c|c|}
\hline \multicolumn{2}{|c|}{ GHOST Spectrograph Parameters } \\
\hline Wavelength range & $363 \mathrm{~nm}-1000 \mathrm{~nm}$ \\
\hline Échelle Grating & $\begin{array}{l}\text { Richardson Grating Labs 424E, master MR234 } \\
65^{\circ} \text { blaze (R-2.14) } \\
\gamma=0.56^{\circ} \\
\mathrm{G}=52.67 \text { grooves } / \mathrm{mm} \\
\text { Ruled area: } 204 \mathrm{~mm} \times 410 \mathrm{~mm}\end{array}$ \\
\hline Collimator & $\begin{array}{l}1764 \mathrm{~mm} \text { f.I. @ f/10 } \\
176 \mathrm{~mm} \text { beam diameter at échelle }\end{array}$ \\
\hline White pupil magnification & 0.45 \\
\hline Beam Splitter & $\begin{array}{l}\text { Cut on wavelength: } 529.4 \mathrm{~nm} \text { ( center of order } 65 \text { ) } \\
\text { Order Overlap: } \pm 1.5 \text { orders } \\
\text { Reflection Band: } \\
\quad \text { R > } 97 \% \text { ( goal } 99 \% \text { ) from } 360 \mathrm{~nm} \text { to } 517.4 \mathrm{~nm} \\
\text { Transmission band: } \\
\quad \text { T > } 96 \% \text { ( goal } 98 \% \text { ) from } 541.4 \mathrm{~nm} \text { to } 1000 \mathrm{~nm} \\
\text { Substrate: } \\
\quad \text { N-BK7HT } 210 \mathrm{~mm} \times 126 \mathrm{~mm} \times 15 \mathrm{~mm}, 5^{\circ} \text { wedge }\end{array}$ \\
\hline Cross dispersers & $\begin{array}{l}\text { Blue: } 1050 \text { lines } / \mathrm{mm} \text { VPHG, } \alpha=35^{\circ} \\
\text { Substrate: Fused Silica } 129 \mathrm{~mm} \times 140 \mathrm{~mm} \times 10 \mathrm{~mm} \times 2 \\
\text { Red: } 500 \text { lines } / \mathrm{mm} \text { VPHG, } \quad \alpha=31^{\circ} \\
\text { Substrate: Fused Silica } 129 \mathrm{~mm} \times 140 \mathrm{~mm} \times 10 \mathrm{~mm} \times 2 \\
\text { Beam Diameter of recorded VPHG: } 127 \mathrm{~mm}\end{array}$ \\
\hline Blue Camera & $\begin{array}{l}\text { Orders: } 95 \text { to } 64 \\
\text { Minimum order width: } 1.12 \times \text { FSR } \\
\text { wavelength range: } 360 \mathrm{~nm} \text { to } 542 \mathrm{~nm} \\
\text { field of view: } 14.3 \text { degrees (full angle) } \\
\text { focal length: } 295 \mathrm{~mm} \\
\text { fastest beam: } \mathrm{f} / 3 \\
\text { (CCD: } 4 \mathrm{kx} 4 \mathrm{k} \text { pixel detector area, } 15 \mu \mathrm{m} \text { pixels ) }\end{array}$ \\
\hline Red Camera & $\begin{array}{l}\text { Orders: } 66 \text { to } 34 \\
\text { Minimum order width: } 1.12 \times \text { FSR } \\
\text { wavelength range: } 517 \mathrm{~nm} \text { to } 1000 \mathrm{~nm} \\
\text { field of view: } 25.7 \text { degrees (full angle) } \\
\text { focal length: } 295 \mathrm{~mm} \\
\text { fastest beam: } \mathrm{f} / 2.8 \\
\text { (CCD: } 6 \mathrm{kx} 6 \mathrm{k} \text { pixel detector area on } 15 \mu \mathrm{m} \text { pixels) }\end{array}$ \\
\hline
\end{tabular}

\section{OPTICAL DESIGN TRADE}

The AAO GHOST spectrograph design was originally based on a modified Kiwispec design ${ }^{2}$ which was a R-4 échelle with 4 arms. The R-4 échelle provided a small beam diameter $(100 \mathrm{~mm})$ and a 3:1 anamorphic factor was used to reduce the pupil at the cross dispersers to $33 \mathrm{~mm}$. This design choice gave small camera optics but the narrow cross disperser bandpasses required four cameras. This design was optimized to the production capabilities of Kiwispec. The current design is the result of a trade study to produce a bench spectrograph design optimized to the capabilities and experience 
at HAA. The key goals in the trade study were to reduce the number of arms in the system as far as possible and to provide sufficiently improved throughput of the bench spectrograph to allow it to be located in the Gemini pier lab rather than on the dome floor, without a net loss in the system efficiency. The motivation for locating the spectrograph in the pier lab was the improved environmental stability.

As a consequence of the image slicing, the spectrograph requires a significant amount of cross dispersion, thus ruling out the possibility of prism cross dispersion. VPHG cross dispersers were the only practical design choice. A two arm design was smallest number of arms possible (without a gradient order blocking filter), thus it was elected to pursue a two arm design. A simple calculation suggested a configuration with a $6 \mathrm{k} \mathrm{x} 6 \mathrm{k}$ red detector and $4 \mathrm{k} \mathrm{x} 4 \mathrm{k}$ blue detector as a goal. With $6 \mathrm{k} \times 6 \mathrm{k}$ detectors now in standard production this was a realistic option.

The optical design trade study began with the process of selecting the most efficient disperser configuration. The échelle selection started with finding the most efficient échelle that will fill as much of the detector width as possible on the reddest order. Equation (1) is a very useful approximation to calculate the grating groove frequency required to fill a specific detector width for an échelle spectrograph. This equation is accurate to $5 \%$ for R-2 to R-4 gratings. The reddest order will be the widest on the detector, thus $\lambda$ used in this equation is 1 um. This equation shows that to fill the width of the $6 \mathrm{kx} 6 \mathrm{k}$ detector with some margin for order overlap a groove frequency of approximately $50 \mathrm{G} / \mathrm{mm}$ is appropriate. Equation (2) shows the order number of the reddest order will be in the mid-thirties.

$$
\begin{gathered}
G \cong \frac{1.86 N_{\text {pixels }}}{k \lambda R} \\
m_{\lambda}=\frac{k R}{N_{\text {pixels }}} \cong \frac{1.86}{\lambda G}
\end{gathered}
$$

where $G$ is the grating groove frequency

$N_{\text {pixels }}$ is the number of pixels across the free spectral range of the order

$k$ is the sampling (number of pixels per slit width)

$\lambda$ is wavelength of the order (normally the reddest order)

$R$ is the Resolving Power $(\lambda / \Delta \lambda)$ of the spectrograph

It is important to understand that order width on the detector is chiefly dependent on groove frequency (it is weakly dependent on échelle R-number). The selection of the grating R-number is less important. In principle, a higher Rnumber échelle will yield smaller optics but it does so at the cost of a greater beam anamorphism from the échelle. This will affect the sampling across the orders, the cost of which is either poor resolution at the order edges or over-sampling in the order centers. With an asymmetric white pupil relay the échelle beam size and R-number can be decoupled from the cross disperser and camera optics sizes. With this in mind, the driving factor in selecting the échelle gratings is groove frequency and diffraction efficiency, if a sufficiently large échelle is available to achieve the required resolution.

The Richardson Grating Labs 424E R-2 (R-2.14 to be exact) échelle with a line frequency of 52.67 grooves $/ \mathrm{mm}$ is a close match and it affords us a choice from three available Masters. A review of the performance data published by Richardson clearly reveals that the MR234 master is the preferred selection. The measured performance for this grating is exceptional and is nearly equal to the theoretical maximum with diffraction efficiencies of $\sim 80 \%$ at order centers. By comparison, the $31.6 \mathrm{G} / \mathrm{mm}$ R-4 MR160 master, one of the most common masters in high-resolution spectrographs has a mean diffraction efficiency of $\sim 66 \%$ at order centers and new master to replace the MR160, the MR263 has an efficiency of $\sim 70 \%$, ten percentage points below the MR234.

The efficiency of an échelle is not simply a matter of the lower blaze angle of the R-2 grating providing a greater efficiency, since the other two masters with the same parameter as the MR234 offer relatively poor performance. The 
most significant factor is the quality of the ruling of the master, which admittedly is sometimes more attributable to 'luck of the draw'. It's always best to base a design on an existing grating rather than to gamble on a new ruling.

The grating is illuminated with a $176 \mathrm{~mm}$ beam diameter, resulting in a slight over illumination of the ruled area of the grating. A small over illumination of the grating is an acceptable trade between resolution and throughput. In the design there is a $\sim 2 \%$ gain in resolution at the cost of a $0.3 \%$ light loss.

The design trade then focused on the optimization of the cross disperser efficiency. With an asymmetric white pupil design the line frequency of the grating cross dispersers can be adjusted by changing the white pupil magnification. A comprehensive investigation was undertaken to determine the optimal cross disperser line frequency. The investigation included Rigorous Coupled Wave Analysis, optimizing the grating design to determine the optimal thickness and modulation at each line frequency for the highest mean efficiency over the operating bandwidth.

The result of the study indicated the mean efficiency over the required GHOST bandwidth of a VPHG cross disperser only varied a few percent as a function of line frequency. We emphasize that the VPHG was optimized for the highest mean efficiency rather than the more typical solution of highest peak efficiency at the center of the band. At low frequencies a thick dichromate layer with low index modulation was found to be optimal. The thickness of the dichromate needed to be sufficient to keep the grating in the Kogelnik-Bragg regime, but not too thick. For high frequencies a thin layer with high index modulation was the optimal solution.

The study did not find a configuration with higher cross disperser mean efficiency but instead showed the range of line frequencies where the thickness and modulation of the grating are able to be fabricated, and prescribed a corresponding range of asymmetric white pupil magnification. The result was a target range of $4001 / \mathrm{mm}$ to $600 \mathrm{l} / \mathrm{mm}$ for the red VPHG line frequencies and $800 \mathrm{l} / \mathrm{mm}$ to $1200 \mathrm{l} / \mathrm{mm}$ for the blue VPHG with a preference for line frequencies above 500 $1 / \mathrm{mm}$ in the red and $1100 \mathrm{l} / \mathrm{mm}$ in the blue.

The final values of $500 \mathrm{G} / \mathrm{mm}$ and $1050 \mathrm{G} / \mathrm{mm}$ were selected to meet the required cross dispersion and VPHG size constraints. The constraints for the VPHG line frequencies are tied to the white pupil magnification. A smaller pupil at the VPHG will give the desired higher line frequency, but this smaller pupil is at odds with a larger white pupil collimator mirror size and lower image quality. The white pupil collimator mirror size is a hard constraint given by existing coating equipment. With this constraint the red line frequencies are within the target range and the blue is just slightly below, but still in the manufacturable range.

The VPHGs were designed with slanted fringes (an alpha of $31^{\circ}$ on the red and $35^{\circ}$ on the blue). An alpha of $30^{\circ}$ introduces anamorphic magnification approximately equal to that of the R-2 échelle. This anamorphic magnification of the VPHGs increases the packing of the orders on the detectors without increasing the size of the camera optics but this requires a slanted fringe VPHG, a technology that is now proven. In the case of the blue VPHG incident angle was increased beyond the $30^{\circ}$ to $35^{\circ}$ to prevent VPHG narcissistic ghosts and VPHG substrate reflection ghosts ${ }^{3}$. For the case of the red VPHG an incident angle of $31^{\circ}$ was sufficient to prevent ghosting. VPHG Recombination ghosts were avoided with the non-Littrow cross disperser configuration.

\section{OPTICAL DESIGN}

The optical design of the spectrograph is shown in Figure 1. The spectrograph is based on a white pupil design with VPHG cross dispersion and two cameras. The White Pupil Relay (WPR) with the gratings and beamsplitter are discussed in this section followed by a description of the camera designs and the imaging performance.

The WPR forms the collimator and pupil re-imager. The white pupil relay is a zero-Petzval sum design to eliminate field curvature at the cross-dispersion grating. The typical design for a white pupil relay is two off-axis paraboloids with a plano fold mirror, but such a configuration introduces field curvature at the cross-dispersion grating. The result is grating aberrations at the detector, in the form that can be approximated as a cylindrically curved field. Several methods have been used to reduce the grating aberration, such as a weak cylindrical power on the fold mirror, a cylindrical field lens in the camera, or a cylindrically curved detector.

The zero-Petzval sum relay introduces no grating aberrations, eliminating the need for a mitigating effort and it greatly improves the image quality of the white pupil relay/cross-disperser system over the alternative solutions of cylindrical power on the fold mirror or cylindrical field lens.The radial velocity errors are also reduced because of the greater isoplanatism over the field with this design. Residual aberrations of the WPR relay are near diffraction-limited. 
The WPR is comprised of an off-axis paraboloid main collimator and re-imager, followed by a convex aspheric fold mirror and an off-axis ellipsoid white pupil collimator mirror. From the échelle to the VPHG the system is an afocal relay with a magnification of 0.45 . This reduces the $176 \mathrm{~mm}$ diameter beam at the échelle to an $80 \mathrm{~mm}$ beam at the cross dispersers. The dichroic beam splitter has been configured in the preferred polarity, with the blue reflected and red transmitted. The cut-on wavelength of the beam splitter is set at the center of order $65(529.4 \mathrm{~nm})$ with an overlap of \pm 1.5 order (approximately a $24 \mathrm{~nm}$ transition zone width).

Slit rotation is well controlled in the design, with a net slit rotation of \pm 1.3 degrees, relative to the average rotation of 2.24 degrees. The dispersers dominate the slit rotation in the system. They are orientated so the slit rotation of the échelle acts in the opposite sense to the slit rotation of the cross-disperser, giving a smaller residual slit rotation. The slit rotation was optimized in the system to include camera distortion as well as perspective distortion effects in the relay. These other distortion effects can result in a significantly worse slit rotation if they are not controlled.

The blue camera design is shown in Figure 2. The design is non-achromatic with a tilted detector ( $\sim 1.8$ degrees) to control color. This greatly simplifies the design and eliminates the need for exotic expensive glasses as compared to achromatic designs with un-tilted detectors. The glasses still must be selected to correct the secondary color to image along the tilted detector. The tilted detector has an additional advantage in eliminating ghost reflections from the detector to the slit and back again, as well as reducing other detector reflection ghosts.

The design is fully spherical with the largest element being $173 \mathrm{~mm}$ diameter. All the glasses are Ohara i-line glasses selected for their high blue internal transmittance. The camera has a PBL35Y/S-FSL5Y air spaced doublet followed by a PBL35Y singlet, then a S-FSL5Y singlet, and lastly a fused silica field flattener which serves as the CCD window. The air spacing in the doublet acts as a Merte surface correcting $5^{\text {th }}$ order spherical aberration in the system, eliminating the need for an aspheric surface but the thickness of the air space must be carefully controlled.

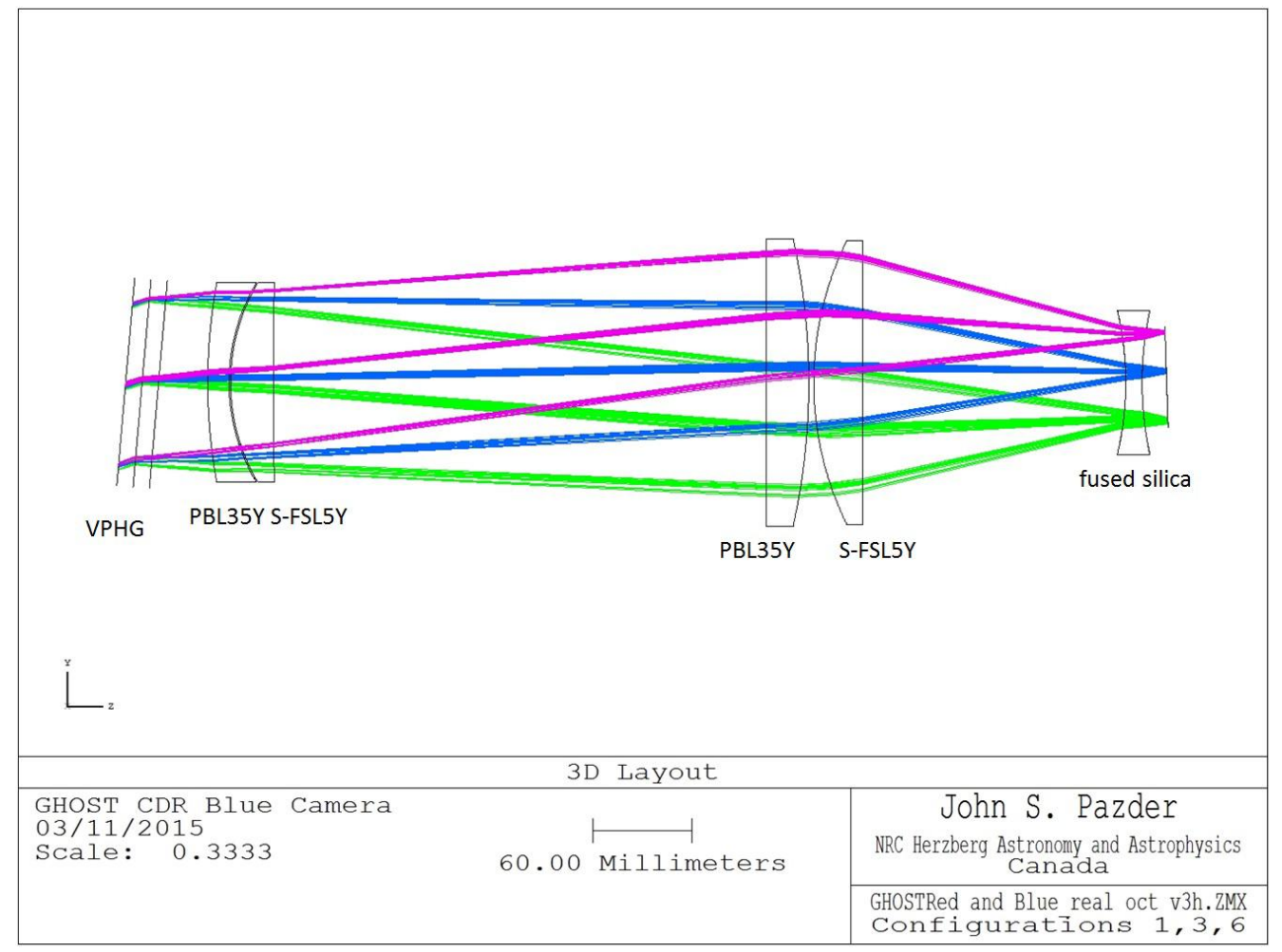

Figure 2. GHOST blue camera layout

The red camera design is shown in Figure 3. The design is also non-achromatic with a tilted detector ( $\sim 2.5$ degrees) to control color. The red camera has a N-BK7HT singlet, then a F2 singlet, then two more N-BK7HT singlets and lastly a fused silica field flattener that serves as the CCD window. The first surface of the first N-BK7HT element is a 4th order aspheric surface. During optimization the aspheric departure was weighted, because otherwise the optimization would 
allow unnecessarily large departures. With the weighting the aspheric departure from a best fit sphere is only $3.8 \mu \mathrm{m}$. The rest of the optical surfaces are spherical. All the glasses have been selected for the highest internal transmittance in the red. The largest element is $219 \mathrm{~mm}$ diameter.

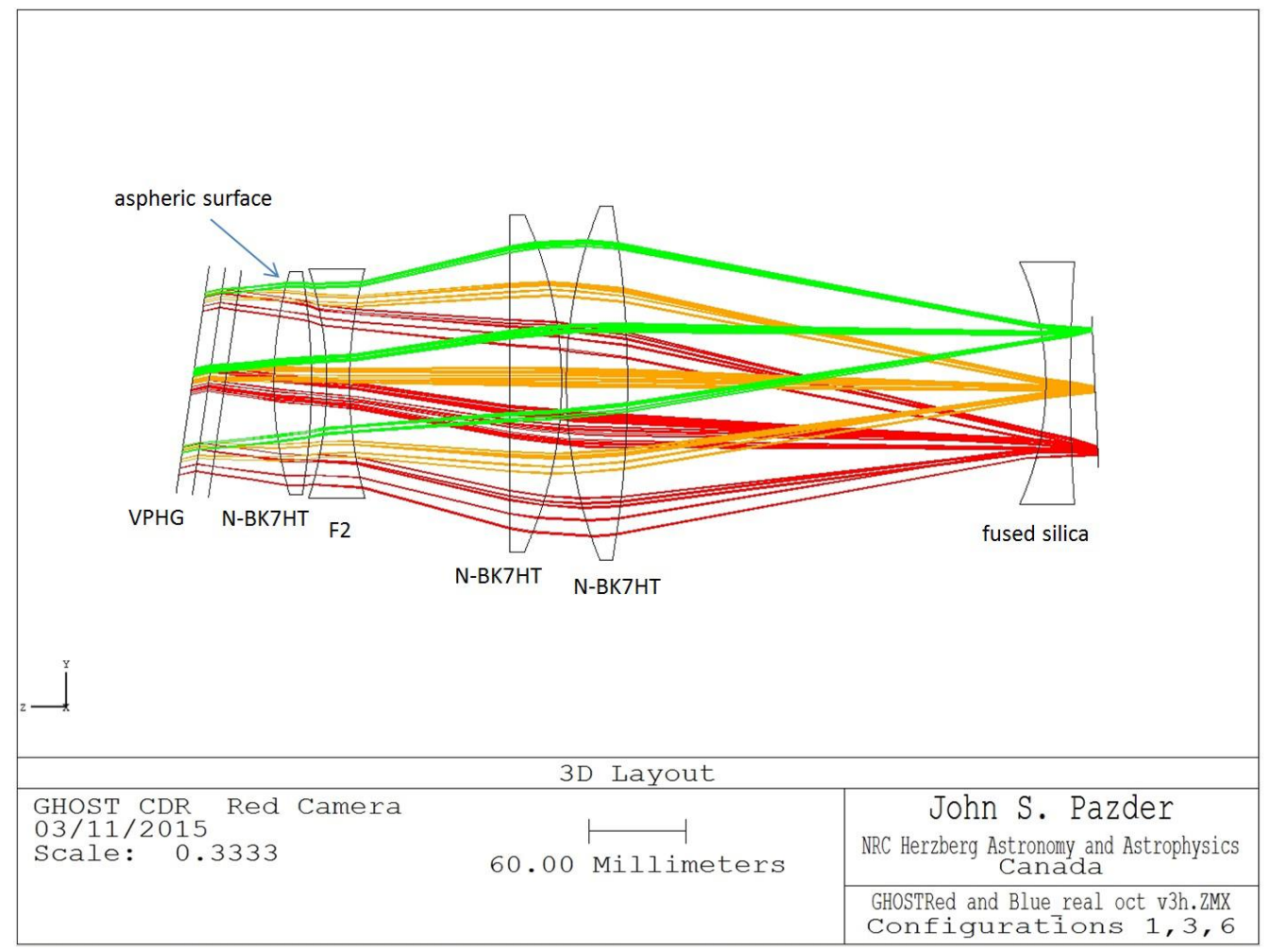

Figure 3. GHOST red camera layout

The spectral formats are shown in the two echellograms in Figure 4. The red camera echellograms (upper panel of Figure 4) show orders 34 to 66 on the $6 \mathrm{k} \mathrm{x} 6 \mathrm{k}, 15 \mathrm{um}$ pixel red detector. Each order is plotted at 1.12 times the free spectral range (FSR), which is the required order coverage for the instrument. In the Figure on the left side the order (m), blaze wavelength $\left(\lambda_{\mathrm{b}}\right)$ in $\mathrm{nm}$, and the Y coordinate of the middle of the order on the CCD in $\mathrm{mm}$ are tabulated. On the right side the free spectral range oversized by 1.12 times $\left(1.12 \Delta_{\mathrm{FSR}}\right)$ in $\mathrm{nm}$, the spectral range to the edge of the CCD in $\mathrm{nm}$, and the ratio of the spectral coverage to $1.12 \mathrm{xFSR}$ are tabulated. As can be seen, $1.12 \mathrm{xFSR}$ of order 34 just fits on the detector.

The blue camera echellograms on the $4 \mathrm{k} \mathrm{x} 4 \mathrm{k}, 15 \mu \mathrm{m}$ pixel blue detector is shown in the lower panel of Figure 4 . The same data as per the red echellograms are tabulated. There is more available detector width on the blue detector but it is 'full' in detector height. The dichoric cut-on wavelength is the center of order 65 , and the \pm 1.5 order overlap can be seen on the echellograms. The transition wavelength between the red and blue cameras $(529.4 \mathrm{~nm})$ was dictated by the height of the blue detector which has resulted in a slightly smaller spectral bandpass on the blue camera than the red camera. This has given the blue arm a slightly greater throughput since the coatings and VPHG perform better on this narrower range. This boost in blue throughput is preferred in meeting the science aims.

Spot diagrams for the red camera (top) and blue camera (bottom) are shown in Figure 5 for the top, middle and bottom orders in each camera. The box size in the spot diagram is $2 \times 2$ pixels, and the circles (in some cases these are elliptical due to beam anamorphism) represent the diffraction limit. It can be seen that the design is close to the diffraction limit for the red orders. The image quality is well within requirement $(80 \%$ ensquared energy within a pixel [15um] for $<$ $870 \mathrm{~nm}$ and $80 \%$ ensquared energy within a box width of 1.5 pixels [22.5um] for $>870 \mathrm{~nm}$ [order 40 and less]). This gives a good margin for fabrication tolerances. 

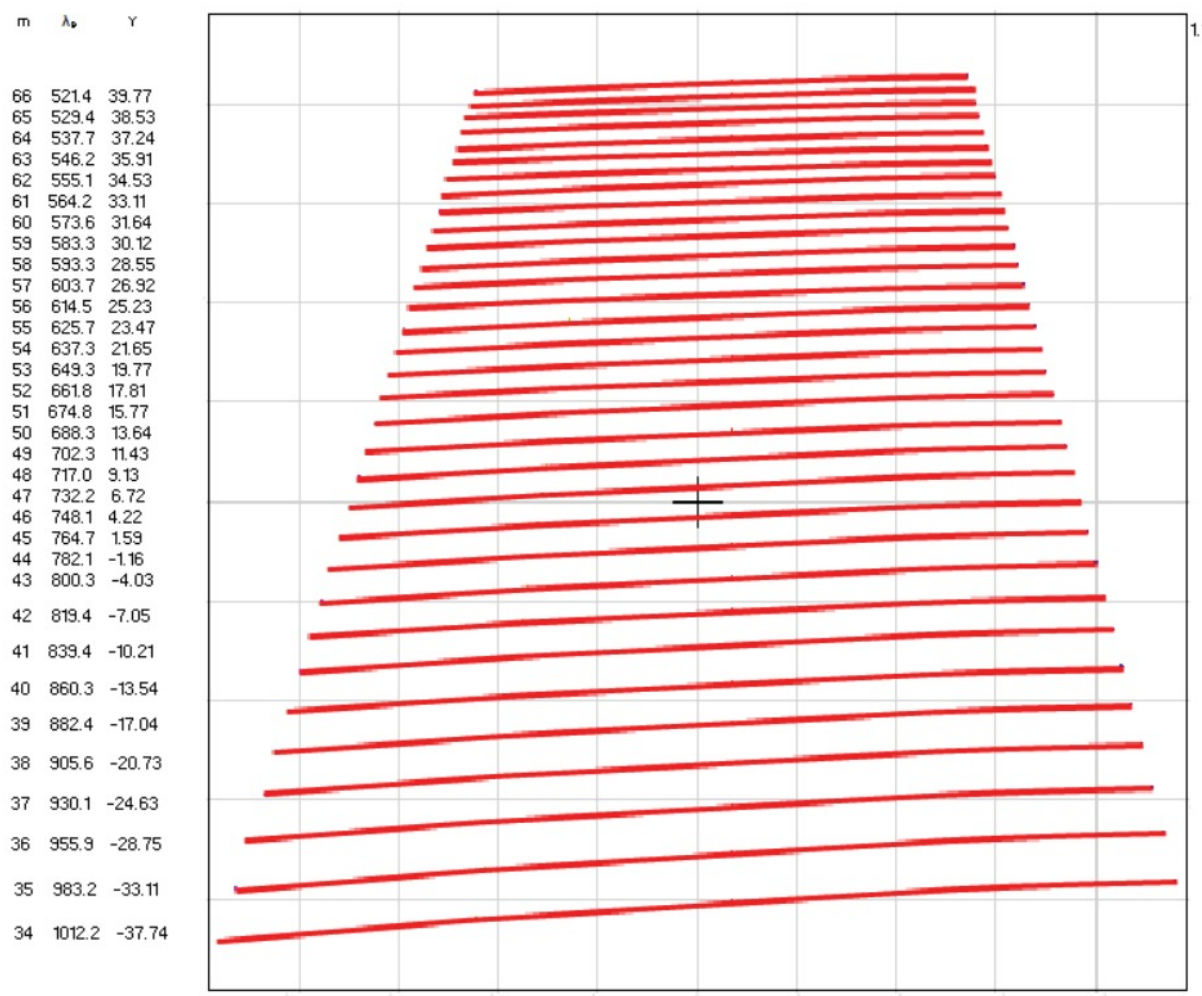

1. $12 \Delta \lambda_{m B} / \Delta \lambda_{C C D}$

8.85 | 17.59 (1.99) 9.12 / 17.86 (1.96) $9.41 / 18.13(1.93)$
$71 ! 18.42(1.90)$ 10.03 / 18.71 (1.87) $10.36 / 19.02(1.84)$ $10.71 / 19.33$ (1.81) 11.07 ! 19.65 (1.78) 11.46 ! 19.98 (1.74) 11.86 ! $20.33(1.71)$ 2.29 / 20.69 (1.68) 2.74 ! $21.06(1.65)$ 13.22 । 21.45 (1.62) 13.72 I 21.85 (1.59) 14.25 / 22.27 (1.56) 14.82 i 22.70 (1.53) $15.42 / 23.16(1.50)$ 16.73 (24.12 (1.44) $16.73>24.12(1.44)$ 18.21125 .16 (1.38) $19.03 / 25.73(1.35)$ 9. 91 i 26.31 (1.32) . $0.85,26.92$ (1.29) 21.85 । 27.57 (1.26) 22.93 ! 28.24 (1.23) $24.09 / 28.95$ (1.20) 25.34 / 29.70 (1.17) $26.69 / 30.48$ (1.14) 28.15 / 31.31 (1.11) 29.74 / 32.18 (1.08) 31.46 / 33.11 (1.05) 33.34 / 34.09 (1.02)
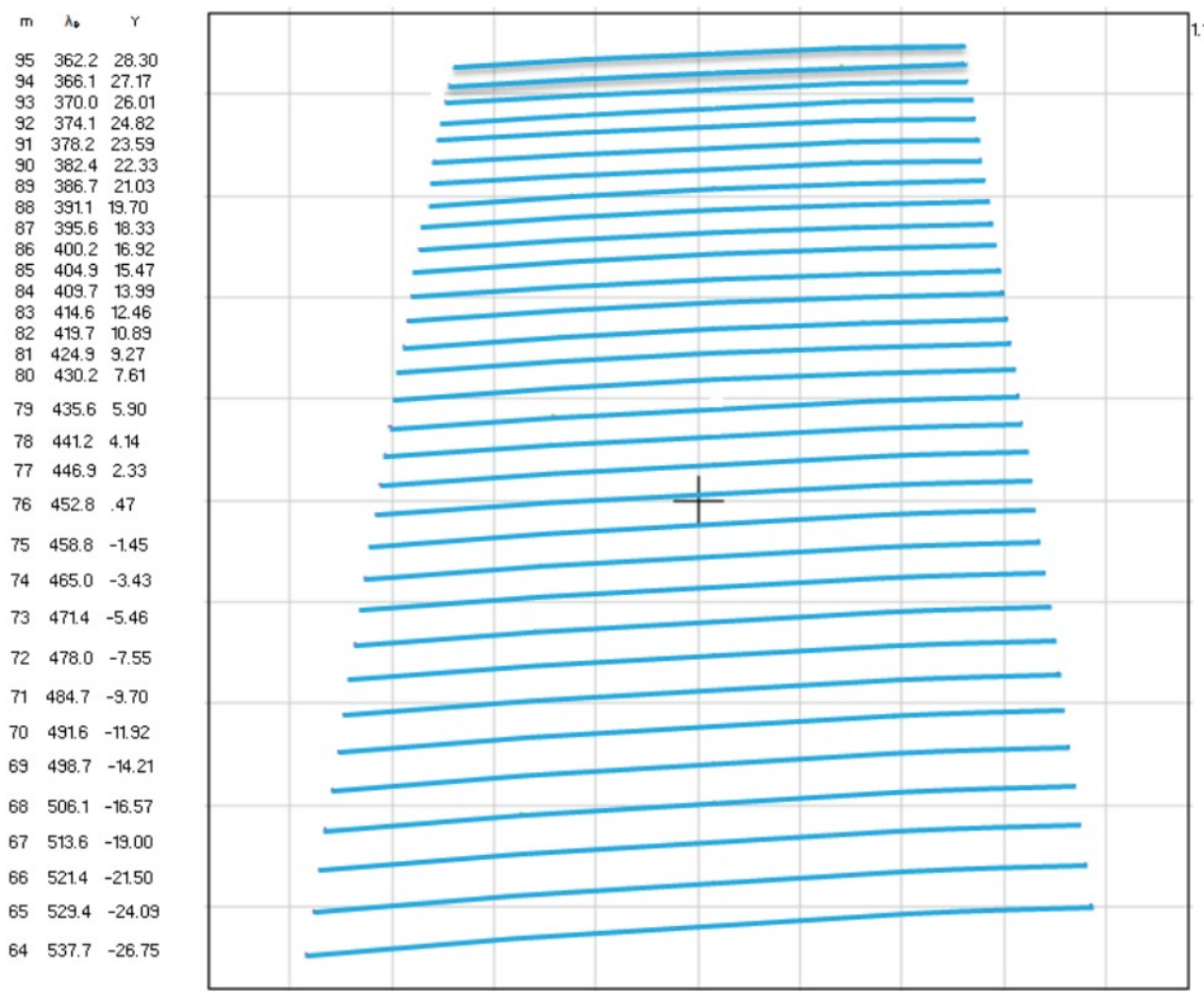

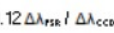
4.2718 .22 (1.92) $4.36 ! 8.36(1.88)$ $4.55+8.44(1.85)$ $4 . 5 5 \longdiv { 8 . 4 4 } ( 1 . 8 5$ $4.65) 8.52(1.83)$ $4.7678 .60(1.81)$ $4.87 / 8.68(1.78)$ $4.98 / 8.77(1.76$ $5.0978 .85(1.74)$ $5.2178 .94(1.72)$

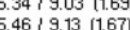
$5.59 / 9.22(1.65)$ 5.73 ! $9.32(1.63)$ $5.88,9.43(1.60)$ $6.0219 .53(1.58)$ $6.18 / 9.64(1.56)$ 6.34 ! $9.75(1.54)$ $6.50 / 9.86(1.52)$ $6.67 / 9.98(1.50)$ $6.85 ? 10.10(1.47)$ $7.04 ! 10.23(1.45)$ $7.23 ! 10.36(1.43)$ $7.44 ! 10.50(1.41)$ $7.65 / 10.64(1.39)$ $7.87 ! 10.78(1.37)$ $8.09 / 10.93(1.35)$ 8.34 / $11.08(1.33)$ $8.59 / 11.24(1.31)$ $8.85 / 11.40(1.29)$ 9.12 / $11.58(1.27)$ $9.41 / 11.76(1.25)$

Figure 4. Red camera (top) and Blue camera (bottom) echellograms 


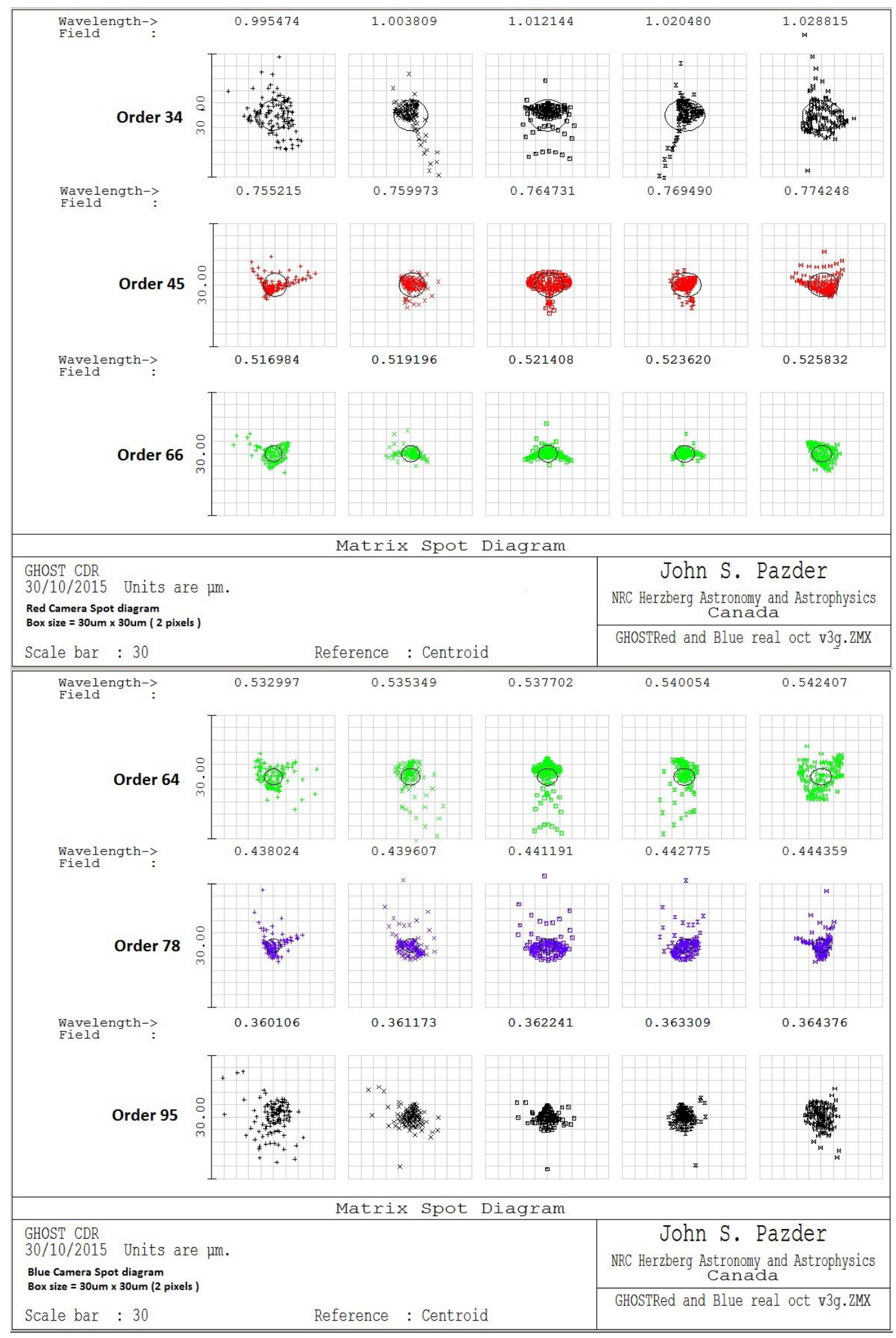

Figure 5. Red camera (top) and Blue camera (bottom) spot diagrams. Boxes are 2 pixels $(30 \mu \mathrm{m})$ and the order width is 1.12xFSR 


\section{SYSTEM THROUGHPUT AND SPECTRAL RESOLUTION}

The bench spectrograph transmittance and throughput is plotted in Figure 6. The transmittance is from the exit of the slit unit to the detector not including the CCD QE while the throughput is the same but with the CCD QE. The échelle diffraction efficiency is for the measured values at the échelle order centers, thus the drop in efficiency across each order is not included in the plot.

The design has been carefully optimized for high transmittance via the coating and glass selection. The white pupil relay mirror will be coated with blue-enhanced protected silver. With the recent advances in protected silver the blue reflectivity is expected to be as high as $95 \%$ at $360 \mathrm{~nm}$, greatly improving the throughput of this spectrograph. The parabolic shape of the VPH grating diffraction efficiency dominates the transmittance profile. On the blue end the dropoff is further accentuated because of the roll-off of the silver coating and glass internal transmittance.

The CCE QE used in the throughput calculation is the as-delivered CCD QE for the E2V devices. The CCD coating is the Astro Multi-2 coating. This coating has excellent QE across the full bandpass, as good as some gradient CCD coatings. The delivered CCD QE met, and at some wavelengths exceeds the 'catalog' QE curve for this coating.

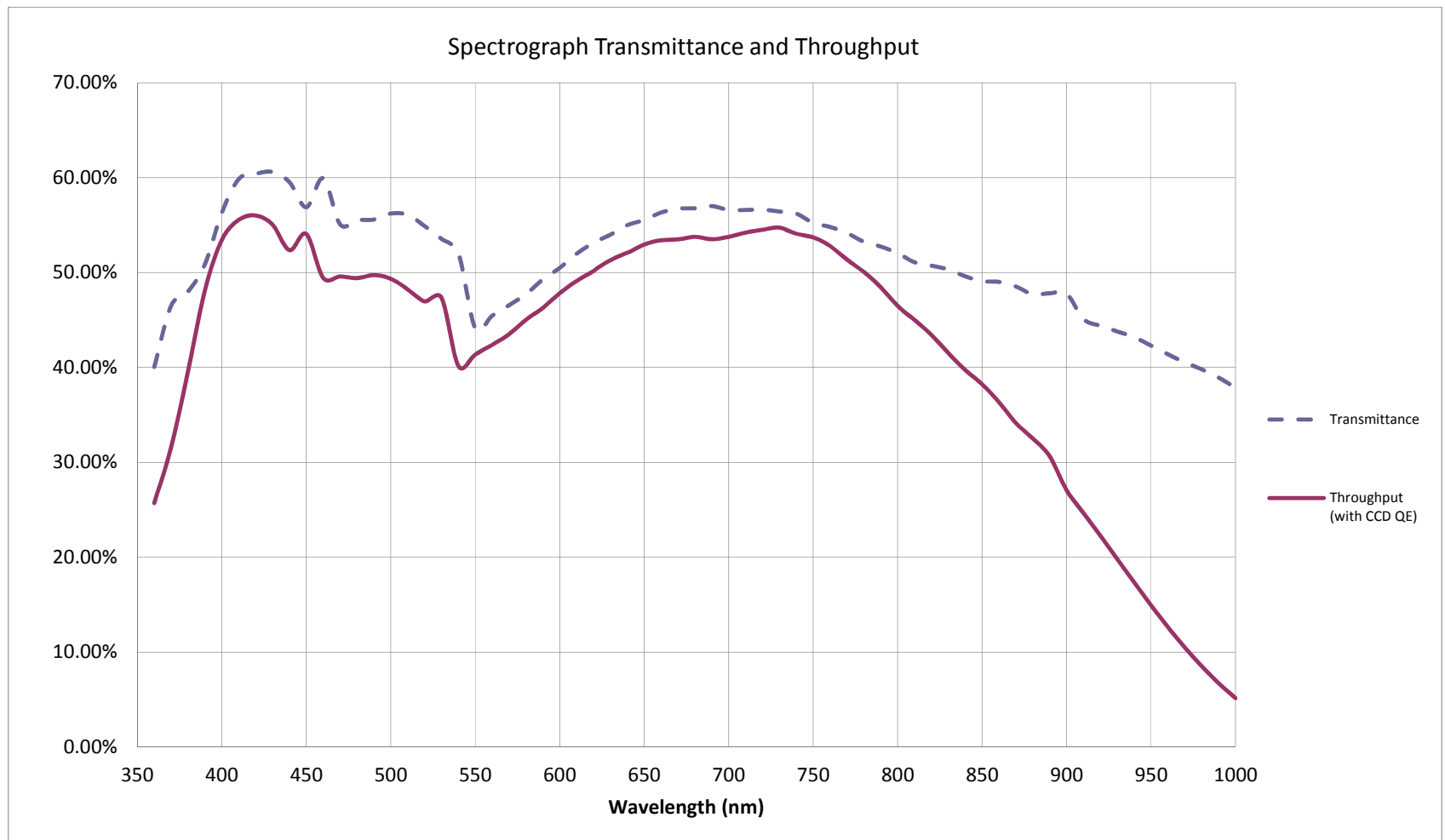

Figure 6. Bench Spectrograph transmittance (from exit of slit unit to detector, not including CCD QE) and throughput (includes CCD QE)

Spectral resolution for the instrument was calculated based on a full-width-half-maximum (FWHM) criterion. The calculation was based on a slit profile model that includes the 19 (for high resolution) or 7 (for standard resolution) micro-lens image profiles. These image profiles included estimated focal ratio degradation. It is interesting to note the focal ratio degradation actually reduced the FWHM of the image profile. These two dimensional slit models were used as the input object for Geometric Image Analysis in the full Zemax model of the spectrograph. Thus all aberrations, magnification effects, slit tilt etc. are fully modeled. The diffraction blurring is not included in such a ray-tracing simulation, but it is not expected to be significant. The geometric analysis was conducted on a grid of $1.5 \mu \mathrm{m}$, ten times finer than the instrument pixel scale, to allow accurate calculation of the image parameters. 
The result of the Geometric Image Analysis were transferred from Zemax to Matlab. The two dimensional images were projected to line images to compute the FWHM. The effects of convolution with the $15 \mu \mathrm{m}$ pixels were included in the calculation. With two slit images corresponding to a known wavelength difference, the reciprocal dispersion can be found and hence the width of the projected profiles can be converted to wavelength units and the resolving power calculated.

The resolving power is actually increased due to the non-zero pixel size and an optical aberration profile. This means that the perfect-optics spectrograph resolutions of 54,200 and 90,400 are actually increased by $\sim 4 \%$ and $\sim 1 \%$ to design resolutions of 56,400 and 91,100, with a resulting near-Gaussian profile. Of course, this could be seen as an artifact of this particular measure (FWHM) of spectrograph resolution - the profile RMS width or many other width measures results in a decrease in resolving power due to the non-zero pixel size and spectrograph optical aberrations ${ }^{4}$.

\section{SUMMARY}

The recent developments in technology, in particular larger and higher QE detectors, slanted fringe VPHG's, nonachromatic camera designs, zero-Petzval white pupil relays and silver coatings have allowed this design to be possible. This design has superb throughput and image quality as compared to the previous generation of spectrographs. The design has passed the final design review in early 2016 and is now in fabrication and is planned to be on-sky in 2018.

\section{ACKNOWLEDGMENTS}

The authors gratefully acknowledge the support from our friends and colleagues in particular Jim Arns at Kaiser Optical Systems for his VPHG advice and calculations and Sarah Shepard at Richardson Gratings/Newport Corporation for her advice on the Échelle grating.

\section{REFERENCES}

[1] Sheinis, A., et al., "The Gemini High-Resolution Optical SpecTrograph (GHOST) Gemini High-Resolution Optical SpecTrograph,” Proc. SPIE 9908, (2016). (this proceedings)

[2] Ireland, M., J., et al., "The AAO's Gemini High-resolution Optical SpecTrograph (GHOST) concept," Pro. SPIE 8446,844629 (2012)

[3] Burgh, E. B., et al., "Recombination Ghosts in Littrow Configuration: Implications for Spectrographs Using Volume Phase Holographic Gratings," PASP 119, 1069-1082 (2007).

[4] Robertson, J. G., "Quantifying resolving power in astronomical spectra," Publ. Astron. Soc. Aust. 30, e048 (2013). 